\title{
Effects of a lifestyle intervention on markers of cardiometabolic risk and oxidized lipoproteins among obese adolescents with prediabetes
}

\author{
Ana Rentería-Mexía ${ }^{1,2}$, Sonia Vega-López ${ }^{2,3}$, Micah L Olson ${ }^{4,5}$, Pamela D Swan ${ }^{2}$, \\ Chong D Lee 2 , Allison N Williams ${ }^{5}$ and Gabriel Q Shaibi ${ }^{2,3,4,5, *}$ \\ 'Departamento de Biotecnología y Ciencias Alimentarias Instituto Tecnológico de Sonora, Obregón, Sonora, \\ México: ${ }^{2}$ School of Nutrition and Health Promotion, Arizona State University, Phoenix, AZ, USA: ${ }^{3}$ Southwest \\ Interdisciplinary Research Center, Arizona State University, USA: ${ }^{4}$ Division of Endocrinology and Diabetes, Phoenix \\ Children's Hospital, Phoenix, AZ, USA: ${ }^{5}$ Center for Health Promotion and Disease Prevention, College of Nursing \\ and Health Innovation, Arizona State University, 500 N. 3rd Street, Phoenix, AZ 85004, USA
}

Submitted 28 April 2018: Final revision received 22 0ctober 2018: Accepted 21 November 2018: First published online 27 December 2018

\begin{abstract}
Objective: Obesity and hyperglycaemia contribute to the atherosclerotic process in part through oxidative modifications to lipoprotein particles. The present study aimed to evaluate the effects of a lifestyle intervention on markers of oxidized lipoproteins in obese Latino adolescents with prediabetes.

Design: Pre-post design.

Setting: Participants were enrolled into a 12-week lifestyle intervention. Measurements pre- and post-intervention included anthropometrics and body composition, lipid panel, oxidized LDL (oxLDL), oxidized HDL (oxHDL), intake of fresh fruits and vegetables, and cardiorespiratory fitness.

Participants: Thirty-five obese Latino adolescents (seventeen females, eighteen males; mean age 15.5 (SD 1.0) years; mean BMI percentile 98.5 (SD 1.2)) with prediabetes.

Results: Intervention participation resulted in significant reductions in weight $(-1.2 \%, P=0.042)$, BMI and BMI percentile $(-2.0$ and $-0.4 \%$, respectively, $P<0.001)$, body fat $(-7.0 \%, P=0.025)$, TAG $(-11.8 \%, P=0.032)$, total cholesterol $(-5.0 \%, P=0.002)$, VLDL-cholesterol $(-12.5 \%, P=0.029)$, and non-HDL-cholesterol $(-6.7 \%, P=0.007)$. Additionally, fitness $(6.4 \%, P<0.001)$ and intake of fruits and vegetables $(42.4 \%, P=0.025)$ increased significantly. OxLDL decreased significantly after the intervention (51.0 (SD 14.0) v. 48.7 (SD 12.8) U/1, $P=0.022$ ), while oxHDL trended towards a significant increase (395.2 (SD 94.6) v. 416.1 (SD 98.4) ng/ml, $P=0.056$ ).

Conclusions: These data support the utility of lifestyle intervention to improve the atherogenic phenotype of Latino adolescents who are at high risk for developing premature CVD and type 2 diabetes.
\end{abstract}

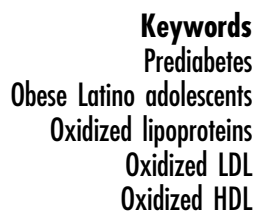

CVD is a leading cause of morbidity and mortality and has its antecedents early in life ${ }^{(1)}$. Obesity, insulin resistance and hyperglycaemia are risk factors for CVD that may contribute to and exacerbate the atherosclerotic process in adolescents $^{(2)}$. Adolescents of Latino descent who are living in the USA are disproportionately impacted by obesity and type 2 diabetes (T2D), both of which contribute to a worsening CVD profile into adulthood ${ }^{(3)}$.

Oxidative stress is a biological mechanism thought to link obesity and T2D with atherosclerosis ${ }^{(4)}$. Oxidative stress generates oxidant species, particularly reactive oxygen species, which are able to oxidize lipoproteins. Oxidation of lipoproteins leads to increased concentrations of oxidized LDL (oxLDL) and oxidized HDL (oxHDL) ${ }^{(5)}$. OxLDL has been associated with CVD and prospectively predicts CVD events $^{(6,7)}$. Data regarding oxHDL are less clear as some experimental studies in cells and animals demonstrate pro-atherogenic properties ${ }^{(8,9)}$, while others suggest antiatherogenic properties ${ }^{(10,11)}$. In human subjects (adolescents and young adults), a cross-sectional study has shown associations between higher concentration of oxHDL and higher BMI and T2D ${ }^{(12)}$, whereas other studies have shown associations of high concentration of oxHDL with young age, low insulin concentration and low waist circumference ${ }^{(13)}$. 
Despite the fact that both oxidized molecules have been independently related to atherosclerosis, very few studies have simultaneously measured oxLDL and oxHDL concentrations in high-risk paediatric populations.

Considering the impact that oxidative stress plays on lipoproteins, there is a need to develop interventions that reduce oxidative stress and promote an anti-oxidative environment early in life. Therefore, the purpose of the present study was to evaluate the effects of a lifestyle intervention on concentrations of oxLDL and oxHDL in obese Latino adolescents with prediabetes, and to investigate the correlations between changes in oxidized lipoproteins and changes in adiposity and lifestyle behaviours.

\section{Methods}

\section{Participants and study design}

This secondary data analysis included thirty-five obese (BMI $\geq 95$ th percentile) Latino adolescents with hyperglycaemia following a 2 -h oral glucose tolerance test. For purposes of the current analysis, elevated post-challenge glucose was defined as a 2 -h glucose $\geq 6.7 \mathrm{mmol} / \mathrm{l}$. The participants (seventeen females, eighteen males; mean age 15.5 (SD 1.0) years) included in the present report were part of a larger, randomized controlled trial of 160 obese Latino adolescents. Details of the parent study ${ }^{(14)}$ along with the effects of the trial on the primary outcomes of insulin sensitivity and quality of life have been reported elsewhere ${ }^{(15)}$. As part of the larger trial, participants who met the American Diabetes Association criteria for prediabetes at baseline were automatically assigned to the intervention group. The rationale for automatically assigning prediabetic adolescents to the intervention included the facts that: (i) prediabetic adolescents can rapidly decompensate and develop T2D (although the intervention was 12 weeks long, the parent study followed participants for 1 year); and (ii) the health disparities focus and community emphasis of the intervention relied heavily on input from community partners and stakeholders who strongly advised against randomizing prediabetic adolescents from a disparity population to a control condition. Given the importance of broad community engagement to the parent research study, there were no prediabetic adolescents in the control programme with whom to compare.

\section{Lifestyle intervention}

The intervention has been described in detail elsewhere ${ }^{(14)}$. Briefly, the 12-week intervention included nutrition education delivered to groups of families (weekly, $60 \mathrm{~min}$ session) and physical activity (weekly, three $60 \mathrm{~min}$ sessions). Nutrition education was delivered by bilingual/bicultural 'promotoras' (community health workers) and focused on health risks of obesity and T2D, increasing consumption of fruits and vegetables, reducing energy from sugar and fat, promoting healthy breakfast and snacking behaviours, and controlling portion sizes. The physical activity sessions were delivered by certified fitness instructors at a local YMCA to groups of eight to ten adolescents and each week included three days of structured and unstructured exercises for $60 \mathrm{~min}$. Structured components included aerobic activities (e.g. running, spinning), anaerobic activities (e.g. athletic drills) and resistance exercises. Unstructured components included team sports and games that promoted social support and bonding among adolescents. Physical activity sessions were designed to elicit an average heart rate of $\geq 150$ beats $/ \mathrm{min}$ for the majority of the session. Heart rate was monitored during sessions using a Polar Heart Rate monitor (Polar USA, Bethpage, NY, USA). The target heart rate was selected based upon previous literature among obese adolescents that demonstrated significant improvements in cardiometabolic health indicators at this intensity level ${ }^{(16)}$.

\section{Anthropometry and body composition assessment}

Participants arrived at the ASU Clinical Research Unit following an overnight fast $(>10 \mathrm{~h})$. Weight was assessed wearing lightweight clothing without shoes and recorded to the nearest $0 \cdot 1 \mathrm{~kg}$ using a balance beam medical scale. Standing height (without shoes) and waist circumference (at the position of the umbilicus) were measured to the nearest $0.1 \mathrm{~cm}$ using a stadiometer and a tape measure, respectively. BMI was calculated as weight divided by height squared, which was used to calculate BMI percentiles based on the Centers for Disease Control and Prevention 2000 growth charts for age and gender. Blood pressure was measured in a relaxed, upright sitting position using an appropriately sized cuff on the right arm after the participant had rested quietly for $5 \mathrm{~min}$ using an Omron IntelliSense HEM-907XL automated monitor (Omron Healthcare, Inc., Bannockburn, IL, USA). Measurements were taken at $1 \mathrm{~min}$ intervals and the average of the three measures was used. Body composition was estimated by bioelectrical impedance analysis (TBF-300A; Tanita Corp of America, Arlington Heights, IL, USA).

\section{Cardiometabolic risk biomarkers: lipid panel and oral glucose tolerance test measurements}

Fasting blood samples $(>10 \mathrm{~h}$ ) were drawn at baseline and after the intervention (within 24-48 h after the last physical activity class) from an antecubital vein. Blood was centrifuged at $3000 \mathrm{rpm}$ at $5^{\circ} \mathrm{C}$ for $15 \mathrm{~min}$, serum/plasma was separated, and aliquots were stored at $-80^{\circ} \mathrm{C}$ until analysis. Total cholesterol, HDL-cholesterol (HDL-C), TAG, LDLcholesterol (LDL-C; calculated by the Friedewald equation $^{(17)}$ ), VLDL-cholesterol (VLDL-C; estimated dividing the TAG value in $\mathrm{mmol} / \mathrm{l}$ by 5 , according to Wilson et al. ${ }^{(18)}$ ) and non-HDL-cholesterol (non-HDL-C; calculated as total cholesterol minus HDL-C) were assessed in serum using standard procedures ${ }^{(19-22)}$. The CV for lipid panel measurements was $<5 \%$.

An oral glucose tolerance test consisting of $75 \mathrm{~g}$ of glucose in solution was administered to measure glucose and insulin 
concentrations at fasting and $2 \mathrm{~h}$. Fasting plasma glucose and 2-h glucose concentrations were determined by the glucose oxidase method using the automated chemistry analyser Cobas C111 (Roche Diagnostics, Indianapolis, IN, ISA) ${ }^{(19,23)}$, while fasting and 2-h insulin concentrations were measured by ELISA (ALPCO Diagnostics, Windham, NH, USA). Insulin resistance was calculated using homeostasis model assessment (HOMA-IR) ${ }^{(24)}$. The CV for glucose and insulin measurements was $<5$ and $<15 \%$, respectively.

\section{Oxidized lipoprotein measurements}

OxLDL and oxHDL were measured using commercially available ELISA kits through sandwich enzyme immunoassay techniques. OxLDL was measured using a kit from Mercodia AB (Uppsala, Sweden) with the same specific murine monoclonal antibody mAb-4E6 as in the assay described by Holvoet et al. ${ }^{(25)}$, with inter- and intra-assay $\mathrm{CV}$ of $1 \cdot 1$ and $2 \cdot 8 \%$, respectively. OxHDL was measured with a proprietary antibody from MyBioSource, Inc. (San Diego, CA, USA), with inter- and intra-assay CV of 2.9 and $8.8 \%$, respectively.

\section{Measurement of behavioural variables}

Dietary intake of fresh fruits and vegetables and cardiorespiratory fitness were evaluated as behavioural variables. Fresh fruit and vegetable intake was assessed using the Block Food Screener for Ages 2-17, version 2007, which was adapted from the Block Kids $2004 \mathrm{FFQ}^{(26)}$. This screener has been used to evaluate dietary intake of children from diverse ages and ethnicities and has been validated by Hunsberger et al. ${ }^{(27)}$. The screener probes the frequency and quantity of food and beverage items that were consumed by the participant during the previous week. The items included were developed from National Health and Nutrition Examination Survey (NHANES) data for adolescents aged 2-17 years. Frequency is determined by the question 'How many days last week did you eat or drink it?', where 'it' refers to each specific food or beverage item. Quantity is determined by the question 'How much in one day?' These data are used to assign age- and sexspecific portion sizes based on amounts consumed in the most recent NHANES survey from $24 \mathrm{~h}$ recall data. Using the reported frequency and quantity data adjusted for established age- and sex-specific portion sizes, the screener can yield a calculated $\mathrm{g} / \mathrm{d}$ intake for fresh fruits and vegetables. Cardiorespiratory fitness $\left(\mathrm{VO}_{2 \max }\right)$ was estimated by a submaximal treadmill test as described by Nemeth et $a l .{ }^{(28)}$. Participants walked on a treadmill at a self-selected speed at $0 \%$ grade for $4 \mathrm{~min}$. The grade was then increased to $5 \%$ while speed was maintained for four more minutes. Heart rate was recorded at the end of the 8 min and entered into a sex- and age-specific prediction equation to estimate $\mathrm{VO}_{2 \max }$.

\section{Statistical analyses}

Untransformed outcome variables were expressed as mean and SD. For skewed variables, inverse or natural log transformations were calculated to meet the normality assumption; otherwise non-normal variables were analysed by the non-parametric Wilcoxon signed-rank test. Differences between pre- and post-intervention outcomes were evaluated using paired-samples $t$ tests for unadjusted models. Baseline differences and changes following the intervention between boys and girls for oxLDL and oxHDL were explored using independent-sample $t$ tests. Within-subject correlations were examined between changes in oxidized lipoproteins as dependent variables and changes in $\mathrm{BMI}$, body fat, $\mathrm{VO}_{2 \max }$ and dietary intake of fresh fruits and vegetables as independent variables, after adjustment for sex. For this latter statistical analysis, the linear mixed models with maximum likelihood estimates were used to compute the correlation matrix and within-subject correlation coefficients, similar to partial correlation estimated by Bland and $\operatorname{Altman}^{(29)}$. All statistical analyses were performed using $P<0.05$ as significant, with the statistical software packages IBM SPSS Statistics version 22.0 and SAS version 9.4.

\section{Results}

Anthropometric, blood pressure and behavioural variables at baseline and after the intervention are shown in Table 1. Mean compliance with intervention sessions was $86.6 \%$, and the mean heart rate over the 12-week intervention was 158.0 (SD 4.3 ) beats $/ \mathrm{min}$. Participation in the intervention resulted in significant reductions in weight $(-1 \cdot 2 \%, P=0 \cdot 042)$, BMI and BMI percentile $(-2 \cdot 0$ and $-0.4 \%$, respectively, $P<0 \cdot 001)$, body fat $(-7 \cdot 0 \%, P=0.025)$ and waist circumference $(-1.9 \%$, $P=0.025$ ), while systolic and diastolic blood pressure did not change significantly. Significant increases were observed in $\mathrm{VO}_{2 \max }(6.4 \%, P<0.001)$ and dietary intake of fresh fruits and vegetables $(42 \cdot 4 \%, P=0 \cdot 025)$.

Cardiometabolic risk biomarkers are presented in Table 2. Significant reductions were observed for 2 -h glucose concentration $(-19 \cdot 2 \%, P<0 \cdot 001)$, fasting $(-17 \cdot 6 \%$, $P=0.008)$ and 2 -h insulin $(-54.9 \%, P<0.001)$ and HOMA-IR $(-19 \cdot 8 \%, P=0 \cdot 015)$. Most fasting serum lipid measurements were significantly decreased, including TAG $(-11.8 \%, P=0.032)$, total cholesterol $(-5.0 \%, P=0.002)$, VLDL-C $(-12.5 \%, P=0.029)$, HDL-C $(-4.0 \%, P=0.022)$ and non-HDL-C $(-6.7 \%, P=0.0007)$.

Changes in oxidized lipoproteins are shown in Fig. 1. OxLDL was significantly decreased after the intervention (51.0 (sD 14.0) v. 48.7 (sD 12.8) U/l, $P=0.022$ ), while a trend towards significance was observed for increase in OxHDL after the intervention (395.2 (SD 94.6) $v$. 416.1 (sD 98.4) $\mathrm{ng} / \mathrm{ml}, P=0.056$ ).

The correlation coefficients using within-subject design adjusted for sex were computed with changes in oxLDL and changes in oxHDL as dependent variables and 
Table 1 Age and changes in anthropometrics, blood pressure and behavioural variables after the lifestyle intervention among thirty-five obese Latino adolescents (eighteen boys, $51 \%$; seventeen girls, $49 \%$ ) with prediabetes*

\begin{tabular}{|c|c|c|c|c|c|c|}
\hline \multirow[b]{2}{*}{ Variable } & \multicolumn{2}{|c|}{ Pre $(n 35)$} & \multicolumn{2}{|c|}{ Post ( $n$ 35) } & \multirow[b]{2}{*}{$\%$ Change } & \multirow[b]{2}{*}{$P$ value } \\
\hline & Mean & SD & Mean & SD & & \\
\hline Age (years) & $15 \cdot 5$ & 1.0 & $15 \cdot 8$ & 1.0 & 1.9 & - \\
\hline Weight (kg) & 97.4 & $16 \cdot 7$ & $96 \cdot 2$ & $17 \cdot 3$ & $-1 \cdot 2$ & 0.042 \\
\hline Height (cm) & $166 \cdot 6$ & 8.2 & $167 \cdot 4$ & 8.6 & 0.5 & $<0.001$ \\
\hline $\operatorname{BMI}\left(\mathrm{kg} / \mathrm{m}^{2}\right)$ & 35.0 & 4.9 & $34 \cdot 3$ & 4.9 & $-2 \cdot 0$ & $<0.001$ \\
\hline BMI percentile & 98.5 & $1 \cdot 2$ & $98 \cdot 1$ & 1.9 & -0.4 & $0.001 \ddagger$ \\
\hline Body fat (\%) & $46 \cdot 0$ & 7.9 & $42 \cdot 8$ & $6 \cdot 8$ & $-7 \cdot 0$ & 0.025 \\
\hline Waist circumference $(\mathrm{cm})$ & $110 \cdot 2$ & $10 \cdot 4$ & $108 \cdot 1$ & $10 \cdot 9$ & -1.9 & 0.025 \\
\hline Systolic blood pressure $(\mathrm{mmHg})$ & $127 \cdot 1$ & $16 \cdot 5$ & $126 \cdot 1$ & $10 \cdot 9$ & -0.8 & 0.696 \\
\hline Diastolic blood pressure $(\mathrm{mmHg})$ & $73 \cdot 7$ & $9 \cdot 3$ & $72 \cdot 2$ & $7 \cdot 0$ & $-2 \cdot 0$ & 0.204 \\
\hline $\mathrm{VO}_{2 \max } \uparrow(\mathrm{ml} / \mathrm{kg}$ per min) & $29 \cdot 7$ & $5 \cdot 0$ & 31.6 & 4.7 & 6.4 & $<0.001$ \\
\hline Absolute $\mathrm{VO}_{2 \max } \dagger(\mathrm{ml} / \mathrm{min})$ & $2906 \cdot 5$ & 575.0 & 3041.1 & $500 \cdot 0$ & 4.6 & 0.003 \\
\hline Fruit and vegetable intake $(\mathrm{g} / \mathrm{d})$ & $116 \cdot 4$ & $97 \cdot 0$ & 165.8 & 91.0 & $42 \cdot 4$ & 0.025 \\
\hline
\end{tabular}

*Data are shown as raw data.

†n 32.

$\ddagger$ Non-parametric Wilcoxon signed-rank test.

Table 2 Changes in cardiometabolic risk biomarkers after the lifestyle intervention among thirty-five obese Latino adolescents (eighteen boys, $51 \%$; seventeen girls, $49 \%)$ with prediabetes*

\begin{tabular}{|c|c|c|c|c|c|c|}
\hline \multirow[b]{2}{*}{ Variable } & \multicolumn{2}{|c|}{ Pre $(n 35)$} & \multicolumn{2}{|c|}{ Post ( $n$ 35) } & \multirow[b]{2}{*}{$\%$ Change } & \multirow[b]{2}{*}{$P$ value } \\
\hline & Mean & SD & Mean & SD & & \\
\hline Fasting glucose $(\mathrm{mmol} / \mathrm{l})$ & 4.8 & 0.4 & 4.7 & 0.4 & $-2 \cdot 1$ & 0.655 \\
\hline 2-h Glucose $(\mathrm{mmol} / \mathrm{l})$ & $7 \cdot 8$ & 0.7 & $6 \cdot 3$ & 1.7 & $-19 \cdot 2$ & $<0.001$ \\
\hline Fasting insulin (pmol/l) & $232 \cdot 7$ & 279.9 & 191.7 & 243.8 & $-17 \cdot 6$ & $0.008 \dagger$ \\
\hline 2-h Insulin (pmol/l) & 2695.4 & $1145 \cdot 2$ & $1216 \cdot 1$ & 969.5 & -54.9 & $<0.001 \ddagger$ \\
\hline HOMA-IR & $8 \cdot 1$ & 9.9 & 6.5 & $8 \cdot 3$ & $-19 \cdot 8$ & $0.015 t$ \\
\hline TAG $(\mathrm{mmol} / \mathrm{l})$ & 1.7 & 0.8 & 1.5 & 0.8 & -11.8 & $0.032 \ddagger$ \\
\hline Total cholesterol $(\mathrm{mmol} / \mathrm{l})$ & 4.0 & 0.8 & 3.8 & 0.8 & -5.0 & 0.002 \\
\hline VLDL-C (mmol/l) & 0.8 & 0.4 & 0.7 & 0.4 & $-12 \cdot 5$ & $0.029 \ddagger$ \\
\hline LDL-C (mmol/l) & $2 \cdot 2$ & 0.6 & $2 \cdot 1$ & 0.6 & -4.5 & 0.119 \\
\hline $\mathrm{HDL}-\mathrm{C}(\mathrm{mmol} / \mathrm{l})$ & 1.02 & 0.2 & 0.97 & 0.2 & $-4 \cdot 0$ & 0.022 \\
\hline Non-HDL-C $(\mathrm{mmol} / \mathrm{l})$ & 3.0 & 0.8 & $2 \cdot 8$ & 0.8 & $-6 \cdot 7$ & 0.007 \\
\hline
\end{tabular}

HOMA-IR, homeostasis model assessment of insulin resistance; VLDL-C, VLDL-cholesterol; LDL-C, LDL-cholesterol; HDL-C, HLDL-cholesterol; non-HDL-C, non-HDL-cholesterol.

${ }^{*}$ Data are shown as raw data.

$\dagger P$ value using inverse-transformed data.

$\ddagger P$ value using log-transformed data.

changes in BMI, body fat, $\mathrm{VO}_{2 \max }$ and intake of fruits and vegetables as independent variables (Table 3). Changes in oxidized lipoproteins following the intervention were not significantly correlated with changes in adiposity or behavioural measures.

\section{Discussion}

The results of the present study suggest that a lifestyle intervention can significantly reduce oxLDL concentration among obese Latino adolescents with prediabetes. In addition, there was a trend for oxHDL concentration to increase following the lifestyle intervention. These changes were coupled with decreases in weight, BMI, waist circumference and percentage body fat, as well as with improvements in markers of lipid panel and glucose metabolism.

Studies have shown that obese children have higher concentrations of oxLDL than normal-weight children, and higher oxLDL concentrations have been significantly associated with elevated BMI, body fat, visceral fat and waist circumference ${ }^{(30)}$. Few studies have focused on a paediatric population at high risk for T2D. Stringer et al. compared oxLDL concentrations in adolescents with T2D with those of either age-, gender- and BMI-matched (obese) adolescents or unmatched normal-weight controls. Concentrations of oxLDL were significantly higher in 

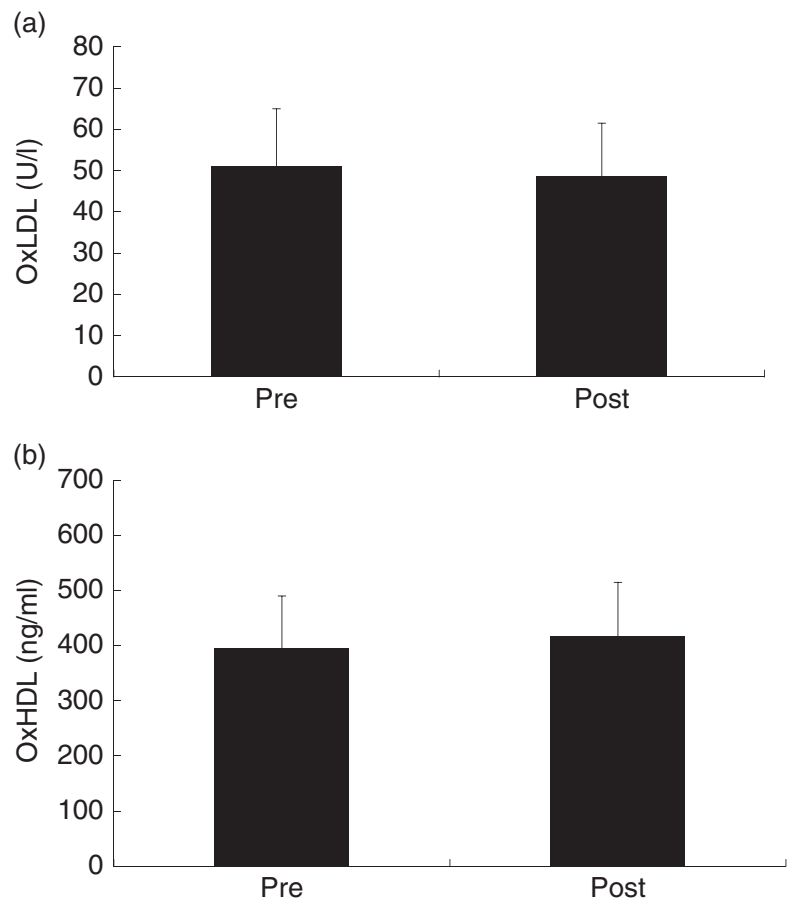

Fig. 1 Oxidized lipoproteins after the lifestyle intervention among thirty-five obese Latino adolescents (eighteen boys, $51 \%$; seventeen girls, $49 \%$ ) with prediabetes; values are means with their standard deviations represented by vertical bars. (a) Oxidized LDL (oxLDL), mean 51.0 (SD 14.0) U/I pre $v$. 48.7 (SD 12.8) U/l post, $P=0.022$; (b) oxidized HDL (oxHDL), mean 395.2 (SD 94.6) $\mathrm{ng} / \mathrm{ml}$ pre v. 416.1 (SD 98.5) $\mathrm{ng} / \mathrm{ml}$ post, $P=0.056$

Table 3 Within-subject correlations between changes in oxidized lipoproteins and changes in adiposity, fitness and fruit and vegetable intake, adjusted for sex, among thirty-five obese Latino adolescents (eighteen boys, 51\%; seventeen girls, $49 \%$ ) with prediabetes

\begin{tabular}{llcc}
\hline $\begin{array}{l}\text { Dependent } \\
\text { variable }\end{array}$ & $\begin{array}{l}\text { Independent } \\
\text { variable }\end{array}$ & $\begin{array}{c}\text { Correlation } \\
\text { coefficient }(r)\end{array}$ & $\begin{array}{c}P \\
\text { value }\end{array}$ \\
\hline Oxidized LDL & BMl & 0.33 & 0.09 \\
& Body fat & 0.05 & 0.80 \\
& VO $2 \max$ & -0.13 & 0.57 \\
Oxidized HDL & Fruit and vegetable intake & -0.23 & 0.22 \\
& BMl & 0.60 & 0.94 \\
& Body fat & -0.03 & 0.89 \\
& VO $_{2 \max }$ & -0.14 & 0.44 \\
& Fruit and vegetable intake & 0.19 & 0.31 \\
\hline
\end{tabular}

T2D and obese adolescents compared with normal-weight controls $^{(31)}$. Similarly, Dasari et al. compared oxLDL concentrations in sedentary adolescents who were healthy weight, obese or type 2 diabetic, and found that oxLDL concentrations were higher in T2D and obese adolescents compared with controls ${ }^{(32)}$. Findings from these crosssectional studies suggest that oxLDL concentration is higher in adolescents with obesity and/or T2D.

Several studies have reported reductions in oxLDL in response to lifestyle intervention among obese adolescents, but none have included adolescents with prediabetes. Kelishadi et al. ${ }^{(33)}$ evaluated the effects of a 6-week lifestyle programme among obese children and showed significant decreases in oxLDL, BMI, waist circumference and percentage body fat. In a previous pilot study among fifteen overweight and obese Latino adolescents with normal glucose tolerance ${ }^{(34)}$, we reported that lifestyle intervention (nutrition and exercise) led to a significant reduction in oxLDL that was independent of changes in weight. In contrast, Kelly et al. ${ }^{(35)}$ reported that 8 weeks of exercise without dietary changes failed to elicit significant change in oxidative stress among nineteen overweight children.

It has been reported that a greater fat mass results in a higher degree of oxidative stress and that reducing adipose depots may result in a decrease in oxidative stress $^{(36,37)}$. Therefore, decreased adiposity and improved glucose regulation may ameliorate the overproduction of oxidative stress biomarkers, as suggested by the lower concentration of oxLDL observed after the lifestyle intervention.

Regarding oxHDL in human subjects, its role in atherogenesis is not clear as the limited number of studies available are contradictory and results remain inconclusive. In cross-sectional studies, obese adult females showed sixfold higher oxHDL concentrations compared with those of normal weight ${ }^{(38)}$, and higher concentrations of oxHDL were reported in obese T2D children compared with their obese and normal-weight peers ${ }^{(12)}$. Conversely, in a crosssectional study of 1395 Finnish adults, oxHDL lipoproteins were inversely associated with age and directly with oxLDL lipoproteins, and additionally in men were associated inversely with insulin, whereas in women with waist circumference. The authors concluded that an elevated risk profile was associated with lower oxHDL concentration ${ }^{(13)}$. In a randomized controlled trial of sedentary women, 6 months of aerobic exercise training significantly increased concentration of oxHDL by $5 \%$ and the ratio oxHDL:HDL by $5 \%$, while participants randomized to the control group exhibited significant decreases in oxHDL and oxHDL:HDL (2 and $1.5 \%$, respectively). Results suggested that oxHDL has an active role in the reverse transport of lipid peroxides ${ }^{(39)}$. These findings along with the observed nonsignificant increase in oxHDL concentration found in the present study may suggest the possibility that oxHDL plays an important role protecting LDL from oxidation or to remove oxidized lipids from LDL.

The anti-atherogenic properties for oxHDL have been proposed to work through different mechanisms, such as the inhibition of TNF- $\alpha^{(10)}$, inhibition of smooth muscle cell proliferation and migration ${ }^{(11)}$, as well as depletion of the regulatory pool of cholesterol available for esterification by acyl-CoA:cholesterol acyltransferase ${ }^{(40)}$. Further, Gao et $a l .{ }^{(41)}$ found that mild HDL oxidation promoted remodelling of human HDL in vitro, which may enhance HDL function including lipid exchange from LDL. In hepatic rodent tissue perfused with HDL containing oxidized lipids (hydroperoxides), a faster removal of oxidized lipids was observed compared with non-oxidized lipids and enhanced reduction of oxidized lipids associated with HDL 
remnants was observed. In contrast, LDL-hydroperoxides were neither removed nor reduced, in support of a possible role for HDL in the detoxification of circulating lipid hydroperoxides ${ }^{(42)}$. Similarly, experiments in rats found an increased selective uptake both in vivo and in vitro of oxidized cholesteryl esters from HDL by hepatic parenchymal cells, compared with non-oxidized cholesteryl esters, and this selective uptake was coupled to a rapid biliary secretion pathway. These results suggest that HDL works as an efficient protection system against potentially atherogenic oxidized cholesteryl ester hydroperoxides ${ }^{(43)}$.

Although animal studies do not necessarily simulate human intervention studies, it is possible that compensatory mechanisms in response to the intervention provoke increases in oxHDL in an attempt to prevent and decrease the atherosclerotic risk promoted by LDL-oxidized lipids and other oxidants ${ }^{(39)}$. Within this hypothesis, it is plausible that oxHDL concentration is a reflection of oxidized lipids that are being removed from LDL as an indication of the lipid peroxide transporting function ${ }^{(44)}$. Data from the present intervention suggest that our lifestyle intervention may reduce the atherogenic risk of obese Latino adolescents through significant reduction in oxLDL. The hypothesis for increased oxHDL concentration after the lifestyle intervention contributing to reduction in oxLDL requires further investigation.

During the present intervention, significant increases in behavioural variables were observed, including fitness and fruit and vegetable consumption. Cross-sectional studies have shown that dietary patterns characterized by a high intake of fruits and vegetables are associated with lower oxidative stress measured by oxLDL, urinary 8-isoprostaglandin F2 $\alpha$ and 8-hydroxy-20-deoxyguanosine ${ }^{(45)}$, as well as higher plasma total antioxidant capacity and glutathione peroxidase activity ${ }^{(46)}$. In adolescents, urinary F2-isoprostane, a marker of oxidative stress, was inversely correlated with intakes of total fruits and vegetables, vitamin C, $\beta$-carotene and flavonoids ${ }^{(47)}$. With regard to interventions, a 1-year dietary intervention that promoted consumption of fruits and vegetables found that plasma antioxidant capacity, measured as oxygen radical absorbance capacity, was significantly correlated with estimated daily intake of total antioxidants from fruits and vegetables ${ }^{(48)}$. In the present intervention, a significant increase in fruit and vegetable intake was observed together with a significant decrease in oxLDL and increase in oxHDL; however, correlations between these changes were not observed.

An important aspect of the present investigation is the potential role of sex and sex hormones on changes in oxidized lipid concentrations during adolescence. It is well established that adult men and women exhibit significant differences in lipoprotein concentrations ${ }^{(49)}$ and lipid metabolism $^{(50)}$. The sexual dimorphism in lipoprotein concentrations emerges early in life where boys and girls exhibit different trajectories for HDL-C and LDL-C throughout adolescence ${ }^{(51)}$. There are also data to suggest that oxidative status differs by sex whereby women may be less susceptible to oxidative stress, which may partially explain sex differences in $\mathrm{CVD}^{(52)}$. There is a dearth of data examining sex differences in markers of oxidative stress in the paediatric population. In our data set, boys and girls were not significantly different at baseline in mean concentration of oxLDL (boys 53.5 (sD 15.4) $\mathrm{U} / 1 v$. girls 48.4 (sD 12.2) $\mathrm{U} / 1 ; P=0.76$ ) or oxHDL (boys 390.3 (sD 88.2) $\mathrm{ng} / \mathrm{ml} v$. girls 400.4 (sD 103.5) $\mathrm{ng} / \mathrm{ml}$; $P=0 \cdot 29)$. While there was no significant sex difference for mean change in oxLDL following the intervention (boys -1.2 (sD 4.7) $\mathrm{U} / 1 v$. girls -3.5 (sD 7.4) $\mathrm{U} / 1 ; P=0.29$ ), oxHDL was significantly more increased in boys than in girls following the intervention (boys 45.1 (SD 77.4) $\mathrm{ng} / \mathrm{ml} v$. girls $-4.7(\mathrm{sD} 66.7) \mathrm{ng} / \mathrm{ml} ; P=0.05)$. Although our study was not designed to examine sex differences in these markers, this interesting observation should be explored further in future lifestyle intervention studies. These studies should also consider whether hormonal changes during development of the menstrual cycle impact lipoprotein metabolism and oxidative stress as these factors were not controlled for in the present analysis.

We acknowledge several limitations of the present analysis that are worthy of comment. First, the lack of a control group limits the generalizability, interpretation and potential application of the results from the study. Furthermore, hyperglycaemia secondary to type 1 diabetes often presents during adolescence, so failure to obtain autoantibodies to distinguish type 1 diabetes risk from T2D risk is a limitation. Another limitation is the daily variability of the lipid panel outcome measures. We attempted to reduce within-person variability through collecting fasting samples and recommending a standard low-fat diet on the day before testing. However, given that post-intervention testing was performed within $24-48 \mathrm{~h}$ after the last intervention session, the potential acute effect of the last exercise bout may have influenced the observed outcomes. An additional limitation was the use of selfreported dietary intake data from an FFQ. Finally, findings from the present study must be taken in the context of the small sample size and the known challenges of accurately assessing changes in dietary intake in adolescents ${ }^{(53)}$.

\section{Conclusion}

In summary, the present intervention showed a significant decrease in oxLDL concentration and a non-significant increase in oxHDL concentration in obese Latino adolescents with prediabetes, coupled with improvements in adiposity and cardiometabolic outcomes. Moreover, behavioural outcomes such as dietary intake of fresh fruits and vegetables and fitness were significantly increased but were not associated with changes in oxidized lipoproteins. Collectively, these findings support the utility of lifestyle intervention to improve the atherogenic phenotype of 
Latino adolescents who are at high risk for developing premature CVD and T2D.

\section{Acknowledgements}

Acknowledgements: The authors thank Ginger Hook, RN, Veronica Zamora, RT, and Mayra Arias-Gastelum, MSc, for their assistance with the study. They are grateful to the children and families who made this study possible. Financial support: This work was supported by the ASU Southwest Interdisciplinary Research Center, an Exploratory Center of Excellence for Health Disparities Research and Training, through a grant from the National Institutes of Health, National Institute on Minority Health and Health Disparities (grant number P20MD002316); and by the ASU Office of Knowledge Enterprise Development, Graduate and Professional Student Association, and the Graduate College. A.R.-M. was supported by the Programa para el Desarrollo Profesional Docente en Educación Superior (PRODEP), México, during her doctoral studies. The funders had no role in the design, analysis or writing of this article. Conflict of interest: None. Authorship: A.R.-M. contributed to the hypothesis development, data acquisition, analysis and interpretation, and manuscript preparation. S.V.-L. contributed to the hypothesis development, data analysis and interpretation, and manuscript revisions. M.L.O. contributed to data interpretation and manuscript revisions. P.D.S. contributed to data interpretation and manuscript revisions. C.D.L. contributed to the study design, statistical analysis, data interpretation and manuscript revisions. A.N.W. contributed to the hypothesis development, data interpretation and manuscript revisions. G.Q.S. contributed to the study design, hypothesis development, funding, supervision, data analysis, data interpretation and manuscript revisions. All authors read and approved the final manuscript. Ethics of buman subject participation: This study was conducted according to the guidelines laid down in the Declaration of Helsinki and all procedures involving human subjects were approved by the Institutional Review Board from Arizona State University. Written parental consent and child assent were obtained prior to any data collection procedures.

\section{References}

1. Strong JP, Malcom GT, McMahan CA et al. (1999) Prevalence and extent of atherosclerosis in adolescents and young adults: implications for prevention from the Pathobiological Determinants of Atherosclerosis in Youth Study. JAMA 281, 727-735.

2. Berenson GS, Srinivasan SR, Bao W et al. (1998) Association between multiple cardiovascular risk factors and atherosclerosis in children and young adults. The Bogalusa Heart Study. N Engl J Med 338, 1650-1656.

3. Skinner AC, Perrin EM, Moss LA et al. (2015) Cardiometabolic risks and severity of obesity in children and young adults. N Engl J Med 373, 1307-1317.
4. Stocker R \& Keaney JF Jr (2004) Role of oxidative modifications in atherosclerosis. Physiol Rev 84, 1381-1478.

5. Fatehi-Hassanabad Z, Chan CB \& Furman BL (2010) Reactive oxygen species and endothelial function in diabetes. Eur J Pharmacol 636, 8-17.

6. Holvoet P, Mertens A, Verhamme P et al. (2001) Circulating oxidized LDL is a useful marker for identifying patients with coronary artery disease. Arterioscler Thromb Vasc Biol 21, 844-848.

7. Meisinger C, Baumert J, Khuseyinova N et al. (2005) Plasma oxidized low-density lipoprotein, a strong predictor for acute coronary heart disease events in apparently healthy, middle-aged men from the general population. Circulation 112, 651-657.

8. Matsunaga T, Hokari S, Koyama I et al. (2003) NF-кB activation in endothelial cells treated with oxidized high-density lipoprotein. Biochem Biophys Res Commun 303, 313-319.

9. Soumyarani VS \& Jayakumari N (2014) Oxidized HDL induces cytotoxic effects: implications for atherogenic mechanism. J Biochem Mol Toxicol 28, 481-489.

10. Girona J, La Ville AE, Heras M et al. (1997) Oxidized lipoproteins including HDL and their lipid peroxidation products inhibit TNF- $\alpha$ secretion by THP-1 human macrophages. Free Radic Biol Med 23, 658-667.

11. Zhou B, Zu L, Chen Y et al. (2017) Myeloperoxidaseoxidized high density lipoprotein impairs atherosclerotic plaque stability by inhibiting smooth muscle cell migration. Lipids Health Dis 16, 3.

12. Marin MT, Dasari PS, Tryggestad JB et al. (2015) Oxidized HDL and LDL in adolescents with type 2 diabetes compared to normal weight and obese peers. J Diabetes Complications 29, 679-685.

13. Kresanov P, Ahotupa M, Vasankari T et al. (2013) The associations of oxidized high-density lipoprotein lipids with risk factors for atherosclerosis: the Cardiovascular Risk in Young Finns Study. Free Radic Biol Med 65, 1284-1290.

14. Williams AN, Konopken YP, Keller CS et al. (2017) Culturallygrounded diabetes prevention program for obese Latino youth: rationale, design, and methods. Contemp Clin Trials 54, 68-76.

15. Soltero EG, Olson ML, Williams AN et al. (2018) Effects of a community-based diabetes prevention program for Latino youth with obesity: a randomized controlled trial. Obesity (Silver Spring) 26, 1856-1865.

16. Ferguson MA, Gutin B, Le NA et al. (1999) Effects of exercise training and its cessation on components of the insulin resistance syndrome in obese children. Int J Obes Relat Metab Disord 23, 889-895.

17. Friedewald WT, Levy RI \& Fredrickson DS (1972) Estimation of the concentration of low-density lipoprotein cholesterol in plasma, without use of the preparative ultracentrifuge. Clin Chem 18, 499-502.

18. Wilson PW, Abbott RD, Garrison RJ et al. (1981) Estimation of very-low-density lipoprotein cholesterol from data on triglyceride concentration in plasma. Clin Chem 27, 2008-2010.

19. Tietz NW (1995) Clinical Guide to Laboratory Tests, 3rd ed. Philadelphia, PA: WB Saunders Company.

20. Rifai N, Warnick GR \& Dominiczak MH (2000) Handbook of Lipoprotein Testing, 2nd ed. Washington, DC: AACC Press.

21. Hatch FT (1968) Practical methods for plasma lipoprotein analysis. Adv Lipid Res 6, 1-68.

22. Stein EA \& Myers GL (1995) National Cholesterol Education Program recommendations for triglyceride measurement: executive summary. The National Cholesterol Education Program Working Group on Lipoprotein Measurement. Clin Chem 41, 1421-1426.

23. Kunst A, Draeger B \& Ziegenhorn J (1984) Methods of Enzymatic Analysis, 3rd ed. vol. VI: Metabolites 1: Carbohydrates, pp. 163-172. Weinheim: Verlag Chemie.

24. Matthews DR, Hosker JP, Rudenski AS et al. (1985) Homeostasis model assessment: insulin resistance and $\beta$-cell function from 
fasting plasma glucose and insulin concentrations in man. Diabetologia 28, 412-419.

25. Holvoet P, Macy E, Landeloos M et al. (2006) Analytical performance and diagnostic accuracy of immunometric assays for the measurement of circulating oxidized LDL. Clin Chem 52, 760-764.

26. NutritionQuest (2007) Block Food Screener for Ages 2-17, version 2007. http://nutritionquest.com/ (accessed August 2018).

27. Hunsberger M, O'Malley J, Block T et al. (2015) Relative validation of Block Kids Food Screener for dietary assessment in children and adolescents. Matern Child Nutr 11, 260-270.

28. Nemeth BA, Carrel AL, Eickhoff J et al. (2009) Submaximal treadmill test predicts $\mathrm{VO}_{2 \max }$ in overweight children. $J$ Pediatr 154, 677-681.

29. Bland JM \& Altman DG (1995) Calculating correlation coefficients with repeated observations: Part 1 - correlation within subjects. BMJ 310, 446.

30. Kelly AS, Jacobs DR Jr, Sinaiko AR et al. (2010) Relation of circulating oxidized LDL to obesity and insulin resistance in children. Pediatr Diabetes 11, 552-555.

31. Stringer DM, Sellers EA, Burr LL et al. (2009) Altered plasma adipokines and markers of oxidative stress suggest increased risk of cardiovascular disease in First Nation youth with obesity or type 2 diabetes mellitus. Pediatr Diabetes 10, 269-277.

32. Dasari PS, Gandomani BS, Teague AM et al. (2016) Glycemic variability is associated with markers of vascular stress in adolescents. J Pediatr 172, 47-55.e2.

33. Kelishadi R, Hashemi M, Mohammadifard N et al. (2008) Association of changes in oxidative and proinflammatory states with changes in vascular function after a lifestyle modification trial among obese children. Clin Chem 54, $147-153$.

34. Ryder JR, Vega-Lopez S, Gaesser GA et al. (2014) Heterogeneous vascular responses to lifestyle intervention in obese Latino adolescents. Metab Syndr Relat Disord 12, 509-515.

35. Kelly AS, Steinberger J, Olson TP et al. (2007) In the absence of weight loss, exercise training does not improve adipokines or oxidative stress in overweight children. Metabolism 56, 1005-1009.

36. Mohn A, Catino M, Capanna R et al. (2005) Increased oxidative stress in prepubertal severely obese children: effect of a dietary restriction-weight loss program. $J$ Clin Endocrinol Metab 90, 2653-2658.

37. Li C, Feng F, Xiong X et al. (2017) Exercise coupled with dietary restriction reduces oxidative stress in male adolescents with obesity. J Sports Sci 35, 663-668.

38. Peterson SJ, Vanella L, Gotlinger K et al. (2016) Oxidized HDL is a potent inducer of adipogenesis and causes activation of the Ang-II and 20-HETE systems in human obese females. Prostaglandins Other Lipid Mediat 123, 68-77.
39. Tiainen S, Luoto R, Ahotupa M et al. (2016) 6-mo aerobic exercise intervention enhances the lipid peroxide transport function of HDL. Free Radic Res 50, 1279-1285.

40. Francis GA, Mendez AJ, Bierman EL et al. (1993) Oxidative tyrosylation of high density lipoprotein by peroxidase enhances cholesterol removal from cultured fibroblasts and macrophage foam cells. Proc Natl Acad Sci U S A 90, 6631-6635.

41. Gao X, Jayaraman S \& Gursky O (2008) Mild oxidation promotes and advanced oxidation impairs remodeling of human high-density lipoprotein in vitro. J Mol Biol 376, 997-1007.

42. Christison J, Karjalainen A, Brauman J et al. (1996) Rapid reduction and removal of HDL- but not LDL-associated cholesteryl ester hydroperoxides by rat liver perfused in situ. Biochem J 314, 739-742.

43. Fluiter K, Vietsch H, Biessen EA et al. (1996) Increased selective uptake in vivo and in vitro of oxidized cholesteryl esters from high-density lipoprotein by rat liver parenchymal cells. Biochem J 319, 471-476.

44. Ahotupa M, Suomela JP, Vuorimaa T et al. (2010) Lipoprotein-specific transport of circulating lipid peroxides. Ann Med 42, 521-529.

45. Cocate PG, Natali AJ, Oliveira A et al. (2014) Fruit and vegetable intake and related nutrients are associated with oxidative stress markers in middle-aged men. Nutrition $\mathbf{3 0}$, 660-665.

46. Hermsdorff HH, Barbosa KB, Volp AC et al. (2012) Vitamin C and fibre consumption from fruits and vegetables improves oxidative stress markers in healthy young adults. Br J Nutr 107, 1119-1127.

47. Holt EM, Steffen LM, Moran A et al. (2009) Fruit and vegetable consumption and its relation to markers of inflammation and oxidative stress in adolescents. J Am Diet Assoc 109, 414-421.

48. Cao G, Booth SL, Sadowski JA et al. (1998) Increases in human plasma antioxidant capacity after consumption of controlled diets high in fruit and vegetables. Am J Clin Nutr 68, 1081-1087.

49. Freedman DS, Otvos JD, Jeyarajah EJ et al. (2004) Sex and age differences in lipoprotein subclasses measured by nuclear magnetic resonance spectroscopy: the Framingham Study. Clin Chem 50, 1189-1200.

50. Mittendorfer B (2005) Sexual dimorphism in human lipid metabolism. J Nutr 135, 681-686.

51. Jolliffe CJ \& Janssen I (2006) Distribution of lipoproteins by age and gender in adolescents. Circulation 114, 1056-1062.

52. Kander MC, Cui Y \& Liu Z (2017) Gender difference in oxidative stress: a new look at the mechanisms for cardiovascular diseases. J Cell Mol Med 21, 1024-1032.

53. Livingstone MB, Robson PJ \& Wallace JM (2004) Issues in dietary intake assessment of children and adolescents. Br J Nutr 92, Suppl. 2, S213-S222. 